\title{
Modelling and researching of forced spatial vibrations of axial fans
}

\author{
Valentin Slavov, ${ }^{1, *}$ and Georgi Vukov $^{2}$ \\ ${ }^{1}$ University of Chemical Technology and Metallurgy, Department of Applied Mechanics, 1756 Sofia, Bulgaria \\ ${ }^{2}$ University of Forestry, Faculty of Forest Industry, 1756 Sofia, Bulgaria
}

\begin{abstract}
This work presents a mechanic - mathematical matrix modelling of the forced spatial vibrations of an axial fan. The axial fan is considered as a mechanical system consisting of three rigid bodies and with 18 degrees of freedom. The differential equations of the forced vibrations are derived. They take into account the mass, inertial, elastic, damping and geometric characteristics of this mechanical system. Algorithms are developed for computer calculating, analysis and synthesis of the design of this axial fan. These algorithms are a prerequisite for achieving the required operational properties of the fan and its compliance with the standards and regulations for vibrations' impact on the human body. Calculations and results of the forced spatial vibrations are provided for specific parameters of the mechanical system.
\end{abstract}

\section{Introduction}

Axial fans are installed in the ventilation systems of industrial, administrative and residential buildings. They drive the fluid into the system, which improves the microclimate in the rooms. Besides, the axial fans are machines that generate vibrations. These vibrations have a negative influence on the human body. Admissible values of the vibrations are regulated by the relevant standards [1]. Assessment of the influence of the vibrations on humans is done separately for the $\mathrm{z}$ axis and for the $\mathrm{x}$ and $\mathrm{y}$ axes, i. e. in the three-dimensional space. That is why spatial vibrations should be investigated. The conditions under which dangerous to human health vibrations rise are determined as a result of these investigations.

Some studies have been carried out and the current state of constructions and methods for modeling and calculation of axial fans are analyzed. It can be concluded that many of the constructions are based on obsolete approximate methods [2-5] which do not allow optimal designing.

Modern means [6-8] and methods of geometric, kinematic and dynamic analysis and synthesis, which are based on mechanic-mathematical matrix methods, have to be used in the designing of axial fans [9-13].

The purpose of this work is to perform a mechanic mathematical modeling in the 3D space of the forced vibrations of axial fans. Formulas and algorithms for analysis and synthesis, which are applicable in designing and investigating of axial fans, should be developed. Geometric, kinematic, mass, inertial, elastic, damping properties and force actions of this mechanical system should be taken into account.

\section{Dynamic model}

The dynamic model is shown in Figure 1.

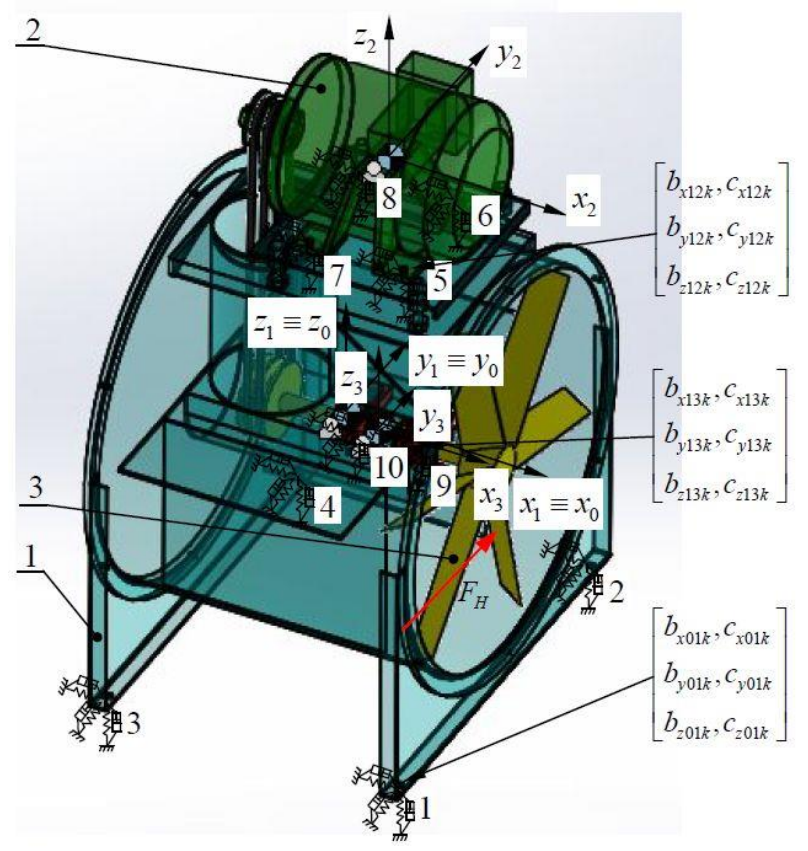

Fig. 1. Dynamic model.

It is assumed that the mechanical system consists of three rigid bodies: 1 - hull, 2 - electric motor and 3 impeller. The hull is mounted to a foundation by means of 4 elastic-damping elements. Their linearized coefficients of elasticity and damping are $c_{x 01 k}, c_{y 01 k}, c_{z 01 k} ; k=1 \div 4, \quad$ and 
$b_{x 01 k}, b_{y 01 k}, b_{z 01 k} ; k=1 \div 4$. The electric motor is also mounted to the hull by means of 4 elastic-damping elements. Their linearized coefficients of elasticity and damping are $c_{x 12 k}, c_{y 12 k}, c_{z 12 k} ; k=5 \div 8$ and $b_{x 12 k}, b_{y 12 k}, b_{z 12 k} ; k=5 \div 8$. The impeller is mounted to the hull via 2 bearings, which are elastic-damping elements, too. Their linearized coefficients of elasticity and damping are $c_{x 13 k}, c_{y 13 k}, c_{z 13 k} ; k=9,10$ and $b_{x 13 k}, b_{y 13 k}, b_{z 13 k} ; k=9,10$. The figure shows the local coordinate systems and the reference coordinate system which coincides with the coordinate system of body 1 and in which all the vectors are projected.

It is assumed that the axes of the local coordinate systems are parallel to the axes of the reference coordinate system. The elastic-damping elements are designated with points from 1 to 10 .

The bodies are characterized by their masses $m_{i}$ and their tensors of mass inertia moments $\mathbf{J}_{\Theta \Theta}^{\mathbf{i}}$.

$$
\mathbf{J}_{\Theta \Theta}^{\mathbf{i}}=\left[\begin{array}{ccc}
J_{O x x} & -J_{O x y} & -J_{O x z} \\
-J_{O y x} & J_{O y y} & -J_{O y z} \\
-J_{O z x} & -J_{O z y} & J_{O z z}
\end{array}\right] ; i=1,2,3
$$

The three bodies of the mechanical system perform spatial vibrations - three small translations and three small rotations relative to the axes of the rectangular local coordinate systems that are immobilly connected to the bodies.

The position of the mechanical system in the space is defined by the generalized coordinate vector which is

$$
\begin{aligned}
& \mathbf{q}=\left[\begin{array}{lllllllll}
x_{1} & y_{1} & z_{1} & \theta_{x 1} & \theta_{y 1} & \theta_{z 1} & x_{2} & y_{2} & z_{2}
\end{array}\right. \\
& \left.\begin{array}{lllllllll}
\theta_{x 2} & \theta_{y 2} & \theta_{z 2} & x_{3} & y_{3} & z_{3} & \theta_{x 3} & \theta_{y 3} & \theta_{z 2}
\end{array}\right]^{\mathrm{T}}
\end{aligned}
$$

The mechanical system has 18 degrees of freedom.

\section{Forced spatial vibrations}

The differential equations which describe the forced vibrations are deduced by using the Lagrange's method.

$$
\frac{d}{d t}\left(\frac{\partial E_{k}}{\partial \dot{\mathbf{q}}}\right)-\left(\frac{\partial E_{k}}{\partial \mathbf{q}}\right)+\frac{\partial E_{D}}{\partial \dot{\mathbf{q}}}+\frac{\partial E_{P}}{\partial \mathbf{q}}=\mathbf{Q}
$$

where $E_{K}$ and $E_{P}$ are respectively the kinetic and the potential energy of the mechanical system, $E_{D}$ is the energy of dissipation or dissipative function and $\mathbf{Q}$ is the vector of generalized forces.

The obtained system of differential equations which describes the forced vibrations of the investigated mechanical system is

$\mathbf{M}_{18 \times 18} \cdot \ddot{\mathbf{q}}_{18 \times 1}+\mathbf{B}_{18 \times 18} \cdot \dot{\mathbf{q}}_{18 \times 1}+\mathbf{C}_{18 \times 18} \cdot \mathbf{q}_{18 \times 1}=$
$=\mathbf{Q}_{18 \times 1}$
The matrix in these equations which characterizes the mass-inertial properties of the mechanical system is $\mathbf{M}$. $\mathbf{B}$ is the matrix that characterizes the damping properties of this system and $\mathbf{C}$ - its elastic properties.

\subsection{Kinetic energy}

The kinetic energy of the mechanical system is calculated by the formula

$$
E_{k}=\sum_{i=1}^{3} E_{K i}
$$

where

$$
\begin{aligned}
& E_{K i}=\frac{1}{2} \cdot\left(\mathbf{m}_{\mathbf{R R}}^{\mathbf{i}} \cdot \mathbf{V}_{\mathbf{C i}}^{\mathbf{0}^{\mathrm{T}}} \cdot \mathbf{V}_{\mathbf{C i}}^{\mathbf{0}}+\mathbf{\Omega}_{\mathbf{i}}^{\mathbf{i}^{\mathrm{T}}} \cdot \mathbf{J}_{\Theta \Theta}^{\mathbf{i}} \cdot \mathbf{\Omega}_{\mathbf{i}}^{\mathbf{i}}\right) ; \\
& \mathbf{m}_{\mathbf{R R}}^{\mathbf{i}}=\int_{V_{i}} \rho_{i} \cdot \mathbf{I} \cdot d V_{i}=m_{i} \cdot \mathbf{I} ; \quad \mathbf{I}=\left[\begin{array}{cccc}
1 & 0 & 0 & 0 \\
0 & 1 & 0 & 0 \\
0 & 0 & 1 & 0 \\
0 & 0 & 0 & 1
\end{array}\right] ;
\end{aligned}
$$

$\mathbf{V}_{\mathbf{C i}}^{\mathbf{0}}$ - vector of the linear velocity of the point $C i$ (the mass center of body $i$ ), projected in the reference coordinate system;

$\boldsymbol{\Omega}_{\mathbf{i}}^{\mathbf{i}}$ - vector of the angular velocity of the body $i$, projected in the local coordinate system.

The elements of the matrix $\mathbf{M}$ are defined by the expression

$$
m_{i, j}=\frac{\partial^{2} E_{K}}{\partial \dot{q}_{i} \cdot \partial \dot{q}_{j}}
$$

\subsection{Potential energy}

The potential energy of the mechanical system is calculated by the formula

$$
\begin{aligned}
E_{P}= & {\left[\sum_{k=1}^{4} \frac{1}{2} \cdot c_{k} \cdot\left(\boldsymbol{\delta} \mathbf{r}_{\mathbf{k}}^{01}\right)^{2}+\sum_{k=1}^{4} \frac{1}{2} \cdot c_{k} \cdot\left(\boldsymbol{\delta} \mathbf{r}_{\mathbf{k}}^{12}\right)^{2}+\right.} \\
& \left.+\sum_{k=1}^{2} \frac{1}{2} \cdot c_{k} \cdot\left(\boldsymbol{\delta} \mathbf{r}_{\mathbf{k}}^{13}\right)^{2}\right]+\left(\sum_{i=1}^{3}-m_{i} \cdot \mathbf{g}^{\mathrm{T}} \cdot \mathbf{R}_{\mathbf{C i}}^{\mathbf{0}}\right)
\end{aligned}
$$

where

$c_{k}$ - elasticity coefficient;

$\mathbf{R}_{\mathbf{C i}}^{\mathbf{0}}$ - vector of the position of the mass center in the reference coordinate system;

$\boldsymbol{\delta} \mathbf{r}_{\mathbf{k}}^{01}$ - the deformation of the elastic elements between the base (denoted conditionally by " 0 ") and body 1 ;

$\boldsymbol{\delta} \mathbf{r}_{\mathbf{k}}^{12}$ - deformation of the elastic elements between body 1 and body 2 ;

$\boldsymbol{\delta} \mathbf{r}_{\mathbf{k}}^{13}$ - deformation of the elastic elements between body 1 and body 3 ;

$\mathbf{g}=\left[\begin{array}{llll}0 & 0 & g & 0\end{array}\right]^{\mathrm{T}}$ is a vector of the gravitational acceleration;

$k$ is the number of the elastic elements between two bodies of the mechanical system.

The elements of the matrix $\mathbf{C}$ are defined by the expression

$$
c_{i, j}=\frac{\partial^{2} E_{P}}{\partial q_{i} \cdot \partial q_{j}}
$$




\subsection{Energy of dissipation (dissipative function)}

Dissipative function is calculated by the formula

$$
F_{B}=\sum \frac{1}{2} \cdot b_{k} \cdot\left(\boldsymbol{\delta} \dot{\mathbf{r}}_{\mathbf{k}}\right)^{2}
$$

where $\boldsymbol{\delta} \dot{r}_{\mathbf{k}}$ is the velocity of deformation of the elastic elements.

The elements of the matrix $\mathbf{B}$ are obtained by replacing the elements of the matrix $\mathbf{C}-c_{i, j}$ with $b_{i, j}$.

$$
B=\left[b_{i, j}\right]
$$

\subsection{Generalized forces}

The presentation of the force $F_{H}$ (Fig. 1) formed by the presence of unbalance is considered below. This force acts in the plane Oyz and has a variable magnitude that is calculated by formula (13). Moreover, the force $F_{H}$ creates a moment about the reference coordinate system that is calculated by formula (14).

The vector of generalized forces has the form

$$
\begin{array}{r}
\mathbf{Q}=\left[\begin{array}{lllllllll}
0 & 0 & 0 & 0 & 0 & 0 & 0 & 0 & 0 \\
0 & 0 & 0 & \mathbf{Q}_{\mathbf{F}(3 \times 1)}{ }^{\mathrm{T}} & \mathbf{Q}_{\mathbf{Q}(3 \times 1)}{ }^{\mathrm{T}}
\end{array}\right]^{\mathrm{T}}
\end{array}
$$

where

$$
\begin{gathered}
\mathbf{Q}_{\mathbf{F}}=\left[\begin{array}{c}
0 \\
F_{H y 3} \\
F_{H z 3}
\end{array}\right] \\
F_{H y 3}=F_{H} \cdot \cos (\omega t) \\
F_{H z 3}=F_{H} \cdot \sin (\omega t) \\
\mathbf{Q}_{\mathbf{Q}}(F)=\mathbf{U}_{\mathbf{i}}^{\mathbf{S 0}^{\mathrm{T}}} \cdot\left(\tilde{\mathbf{r}}_{\mathbf{P i}}^{\mathbf{0}^{\mathrm{T}}} \cdot \mathbf{Q}_{\mathbf{F}}\right) \\
\mathbf{U}_{\mathbf{i}}^{\mathbf{\Omega 0}}{ }^{\mathrm{T}}=\left[\begin{array}{ccc}
1 & 0 & \theta_{y 1} \\
0 & 1 & -\theta_{x 1} \\
0 & \theta_{x 1} & 1
\end{array}\right] \\
\tilde{\mathbf{r}}_{\mathbf{P i}}^{\mathbf{0}^{\mathrm{T}}}=\left[\begin{array}{ccc}
0 & l_{P i z}^{0} & -l_{P i y}^{0} \\
-l_{P i z}^{0} & 0 & l_{P i x}^{0} \\
l_{P i y}^{0} & -l_{P i x}^{0} & 0
\end{array}\right]
\end{gathered}
$$

\subsection{Solutions of the differential equations}

The general solutions of the system of differential equations in matrix form, with initial conditions $t=0, q(0)=q_{0}, \dot{q}(0)=\dot{q}_{0}$ and harmonic disturbing forces

are

$$
\begin{aligned}
q(t) & =\sum_{r=1}^{18} \frac{2}{g_{r}{ }^{2}+h_{r}{ }^{2}}\left[\mathbf{G}_{\mathrm{r}} \cdot \mathbf{M} \cdot \dot{q}(0)+\left(-\alpha_{r} \cdot \mathbf{G}_{\mathrm{r}} \cdot \mathbf{M}+\right.\right. \\
& \left.\left.+\beta_{r} \cdot \mathbf{H}_{\mathrm{r}} \cdot \mathbf{M}+\mathbf{G}_{\mathrm{r}} \cdot \mathbf{B}\right) \cdot q(0)\right] \cdot e^{-\alpha_{r} \cdot t} \cdot \cos \left(\beta_{r} \cdot t\right)+ \\
& +\sum_{r=1}^{18} \frac{2}{g_{r}{ }^{2}+h_{r}{ }^{2}}\left[\mathbf{H}_{\mathrm{r}} \cdot \mathbf{M} \cdot \dot{q}(0)+\left(-\alpha_{r} \cdot \mathbf{H}_{\mathrm{r}} \cdot \mathbf{M}-\right.\right. \\
& \left.\left.-\beta_{r} \cdot \mathbf{G}_{\mathrm{r}} \cdot \mathbf{M}+\mathbf{H}_{\mathrm{r}} \cdot \mathbf{B}\right) \cdot q(0)\right] \cdot e^{-\alpha_{r} \cdot t} \cdot \sin \left(\beta_{r} \cdot t\right)+ \\
& +\operatorname{Re}\left\{\sum_{k=0}^{n} \sum_{r=1}^{18} \frac{2}{g_{r}{ }^{2}+h_{r}{ }^{2}} \cdot\right. \\
& \left.\cdot \frac{\alpha_{r} \cdot \mathbf{G}_{\mathrm{r}}+\beta_{r} \cdot \mathbf{H}_{\mathrm{r}}+i \cdot k \cdot \Omega \cdot \mathbf{G}_{\mathrm{r}}}{\omega_{r}{ }^{2}-k^{2} \cdot \Omega^{2}+i \cdot 2 \cdot k \cdot \sigma_{r} \cdot \omega_{r} \cdot \Omega} \cdot \mathbf{Q} \cdot e^{i \cdot k \cdot \Omega \cdot t}\right\}
\end{aligned}
$$

where

$$
\begin{aligned}
g_{r}= & -2 \cdot \alpha_{r}\left(\mathbf{V}_{r}{ }^{\mathrm{T}} \cdot \mathbf{M} \cdot \mathbf{V}_{r}-\mathbf{W}_{r}{ }^{\mathrm{T}} \cdot \mathbf{M} \cdot \mathbf{W}_{r}\right)- \\
- & 4 \cdot \beta_{r} \cdot \mathbf{V}_{r}^{\mathrm{T}} \cdot \mathbf{M} \cdot \mathbf{W}_{r}+\mathbf{V}_{r}^{\mathrm{T}} \cdot \mathbf{B} \cdot \mathbf{V}_{r}-\mathbf{W}_{r}^{\mathrm{T}} \cdot \mathbf{B} \cdot \mathbf{W}_{r} ; \\
h_{r}= & 2 \cdot \beta_{r}\left(\mathbf{V}_{r}^{\mathrm{T}} \cdot \mathbf{M} \cdot \mathbf{V}_{r}-\mathbf{W}_{r}^{\mathrm{T}} \cdot \mathbf{M} \cdot \mathbf{W}_{r}\right)- \\
& -4 \cdot \alpha_{r} \cdot \mathbf{V}_{r}^{\mathrm{T}} \cdot \mathbf{M} \cdot \mathbf{W}_{r}+2 \cdot \mathbf{V}_{r}^{\mathrm{T}} \cdot \mathbf{B} \cdot \mathbf{W}_{r} ; \\
\mathbf{G}_{r}= & g_{r} \cdot \mathbf{L}_{r}+h_{r} \cdot \mathbf{R}_{r} ; \\
\mathbf{L}_{r}= & \mathbf{V}_{r} \cdot \mathbf{V}_{r}^{\mathrm{T}}-\mathbf{W}_{r} \cdot \mathbf{W}_{r}^{\mathrm{T}} \\
\mathbf{H}_{r}= & h_{r} \cdot \mathbf{L}_{r}-g_{r} \cdot \mathbf{R}_{r} ; \\
\mathbf{R}_{r}= & \mathbf{V}_{r} \cdot \mathbf{W}_{r}^{\mathrm{T}}+\mathbf{W}_{r} \cdot \mathbf{V}_{r}^{\mathrm{T}}
\end{aligned}
$$

\section{Results and discussion}

A dynamic synthesis of a particular axial fan has been performed.

Mass, inertial and geometric parameters of details and units of a particular axial fan are determined by means of a CAD program.

Table 1 gives the mass-inertial, elastic and damping characteristics, the geometric parameters of the mechanical system as well as the disturbing force and the coordinates of the point where it is applied.

Some numerical calculations are performed with these parameters. The purpose of these studies is to select such characteristics of the damping elements of the fan fastening that can minimize the damping time of the vibrations and amplitudes of forced vibrations to be less than those allowed in the standard.

A program has been developed in a mathematical software environment for calculating the forced vibrations of this mechanical system.

A calculation of the forced vibrations of fan with specific parameters is done by the created program.

Analytical and graphical results are obtained.

Figure 2 shows graphical results for the forced vibrations on the generalized coordinates $q_{1}, q_{2}, q_{3}$, which represent the spatial vibrations of body 1 (hull) on the three axes of the local coordinate system. These are the vibrations transmitted by the fan to the building through the foundation. 
Table 1. Parameters of the mechanical system.

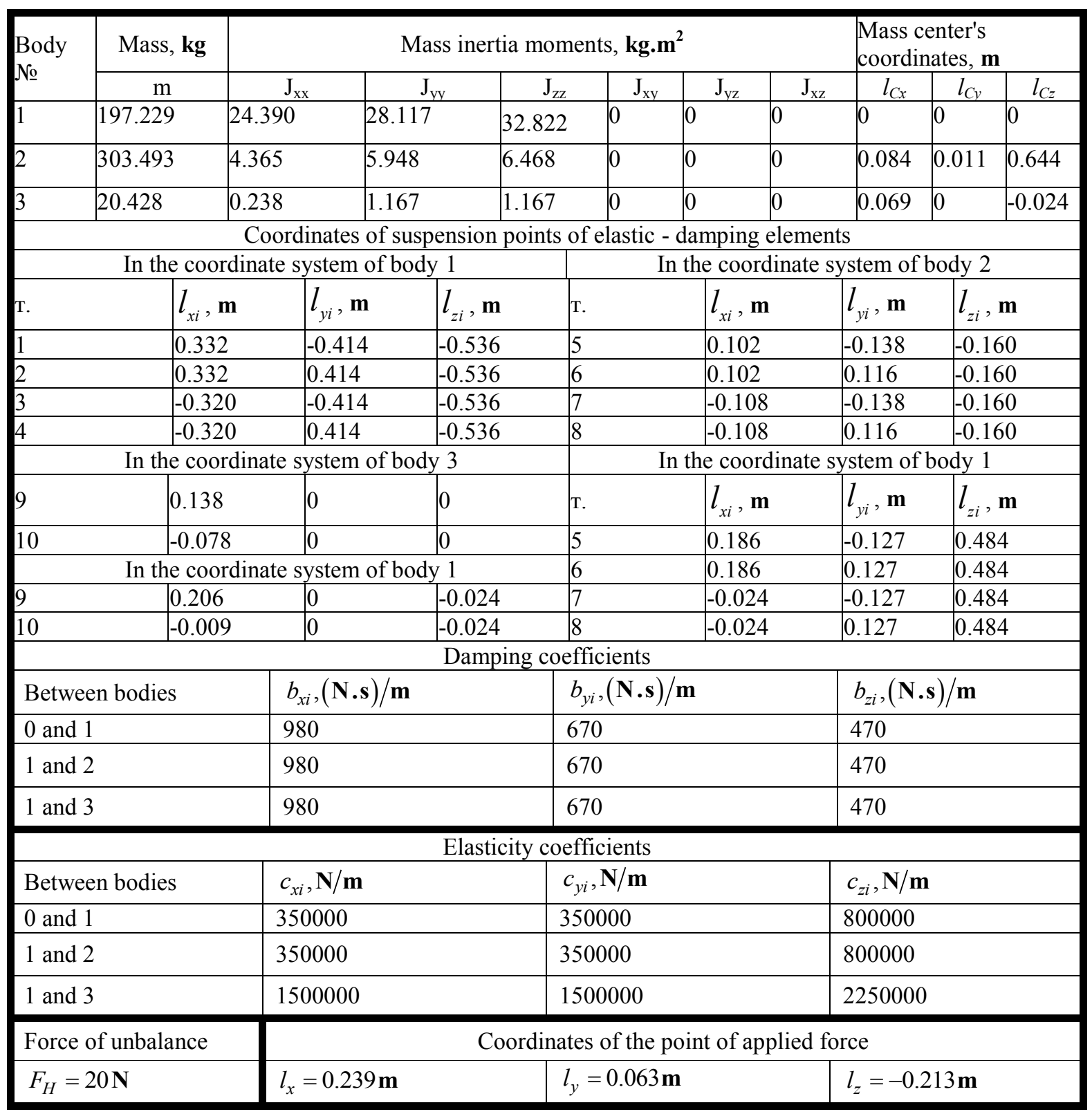



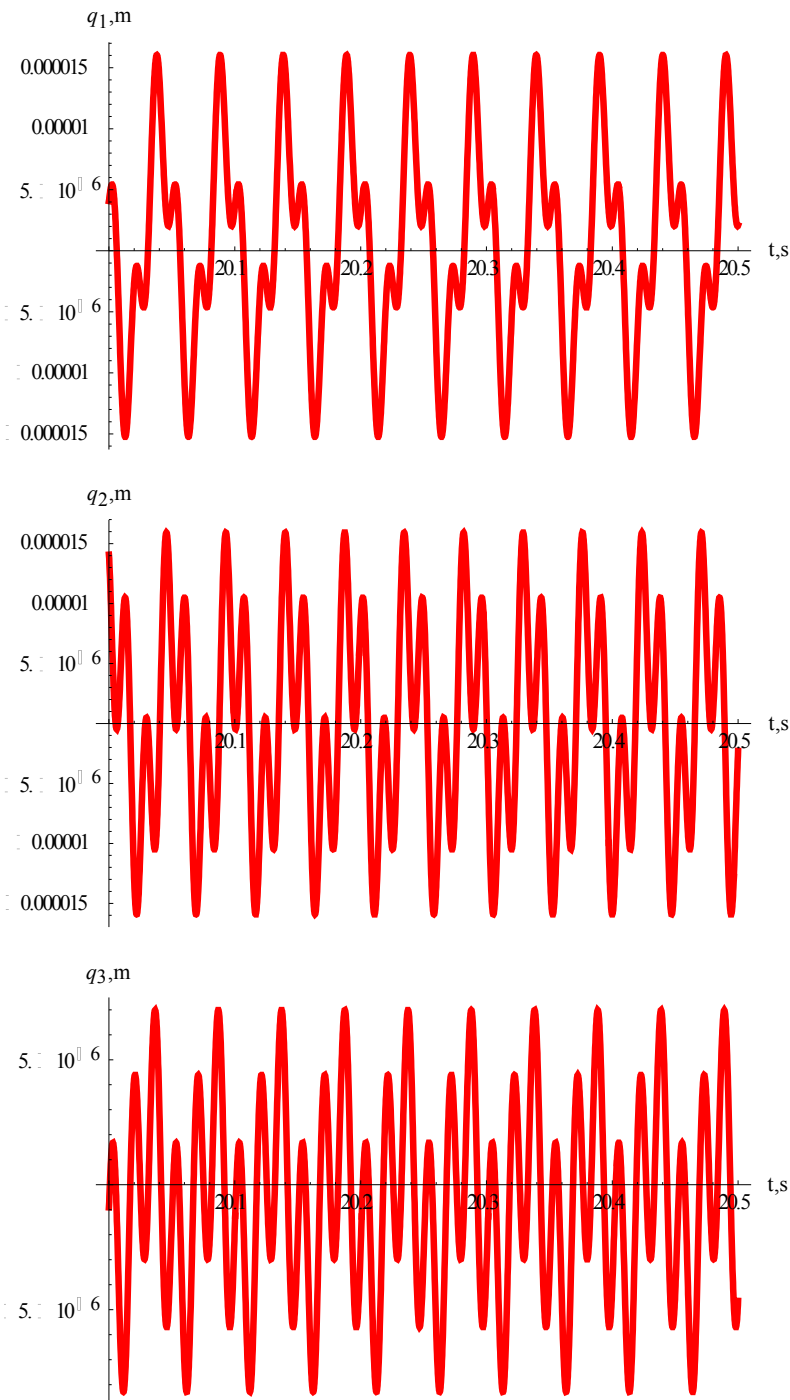

Fig. 2. Forced vibrations of the studied fan.

The analysis of the obtained results shows that the amplitudes of the forced vibrations are within the limits allowed by the standard.

\section{Conclusion}

In the presented paper the differential equations of the forced vibrations of axial fan are deduced. The results obtained in the study of kinematics, dynamics, free undamped vibrations and free damped vibrations of this fan are used. The mass-inertial, elastic and damping characteristics, the geometric parameters of the mechanical system as well as the disturbing force are taken into account. Analytical solutions have been received. Some numerical calculations are performed with the parameters of a concrete fan. The obtained results are presented graphically and they are analyzed.

\section{References}

1. ISO 2631-1:1997. Mechanical vibration and shockEvaluation of human exposure to whole-body vibration-Part 1: General requirements

2. G. Argentini, Simulating a Fan for Industrial Ventilation, Proceedings of the COMSOL Conference, Paris, (2010)

3. G. Kergourlay, S. Kouidri, G. W. Rankin, R. Rey, Experimental investigation of the 3D unsteady flow field downstream of axial fans, FMI 17, 303-314, (2006)

4. J. Hyvärinen, Generalized Methods for Aeroelastic Analysis, RIT, (2003)

5. N. Leso, J. Puttonen, E. Porkka, The effect of foundation on fan vibration response, JSM, 44, 120, (2011)

6. Software product: Solid Works

7. I. Angelov, V. Slavov, Matrix Mechanics. Symbol Programming, (Sofia, Avangard Prima, 2014) (in Bulgarian)

8. Wolfram S. Mathematica: A System for Doing Mathematics by Computer. Reading, Addison Wesley, (1988)

9. I. Angelov, Matrix Mechanics. Dynamics, (Sofia, Avangard Prima, 2011) (in Bulgarian)

10. I. Angelov, Matrix Theory of Vibration in Engineering, (Sofia, Avangard Prima, 2012) (in Bulgarian)

11. I. Angelov, V. Slavov, Matrix Mechanics. Dynamics and Vibrations, Problems, (Sofia, Avangard Prima, 2010) (in Bulgarian)

12. V. Slavov, G. Vukov, Free spatial vibrations of axial fan, SEE, 3, (1), 29-34, (2018)

13. V. Slavov, G. Vukov, Free damped spatial vibrations of axial fan, SEE, 3, (1), 35-41, (2018) 